Published in final edited form as:

J Oral Maxillofac Surg. 2013 July ; 71(7): 1209-1216. doi:10.1016/j.joms.2013.01.028.

\title{
Intraosseous Leiomyosarcoma of the Mandible: A Case Report
}

\author{
Ketan Patel, DDS, PhD ${ }^{\star}$, Christopher French, DDS ${ }^{\dagger}$, Samir S. Khariwala, MD $\ddagger$, Michael \\ Rohrer, DDS, MS§, and Deepak Kademani, DMD, MD\| \\ *Fellow, Oral Head and Neck Oncologic Surgery, Department of Oral and Maxillofacial Surgery \\ †Surgeon, St Paul Oral Surgery, Minneapolis-St Paul, MN \\ ${ }^{\ddagger}$ Assistant Professor, Department of Otolaryngology-Head and Neck Surgery \\ §Professor, Division of Oral and Maxillofacial Pathology \\ "Associate Professor, Fellowship Director, Oral Head and Neck Oncologic Surgery, Department of \\ Oral and Maxillofacial Surgery
}

\begin{abstract}
Leiomyosarcomas are rare smooth muscle tumors that can occur anywhere in the body. These tumors rarely occur in the head and neck owing to the limited amount of smooth muscle in the region. The clinical diagnosis of leiomyosarcoma is challenging because of the nonspecific presentation of the disease. The most definitive diagnosis is based on tissue biopsy or surgical resection and histopathologic confirmation. A case of intraosseous leiomyosarcoma of the mandible and a review of the literature are presented.
\end{abstract}

Smooth muscle tumors are commonly found in the gastrointestinal and genitourinary tracts and retroperitoneum and account for $7 \%$ of all soft tissue sarcomas. ${ }^{1-3}$ Of these, only $4 \%$ occur in the head and neck. ${ }^{4}$ Leiomyomas and leiomyosarcomas are rarely found in the oral cavity and their presence as an isolated intramedullary lesion in the mandible is an even rarer event. The tongue and palate are the most common sites for the presence of leiomyosarcomas in the oral cavity. Other sites in the head and neck that can be involved include the skin of the face and neck, commonly referred to as cutaneous leiomyosarcomas.

The literature suggests that these tumors are often a disease of the elderly, with an average age older than 50 years. There are approximately 70 leiomyosarcomas of the oral cavity reported in the literature. ${ }^{5,6}$ Primary intraosseous lesions are less common owing to the relative paucity of smooth muscle within the maxillofacial skeleton. The authors report an unusual case of a primary intraosseous leiomyosarcoma in the mandible. To date, there are very few reported cases of primary leiomyosarcomas arising in the mandible. The largest case series reported to date was by Vilos et $\mathrm{al}^{6}$ who reported 14 cases of mandibular leiomyosarcoma and 8 cases of maxillary leiomyosarcoma. However, the investigators did not clearly establish if these cases were of primary intraosseous origin or soft tissue

Address correspondence and reprint requests to Dr Kademani: Department of Oral and Maxillofacial Surgery, University of Minnesota Medical Center and School of Dentistry, Minneapolis, MN 55455; kadem001@umn.edu. 
infiltration into the adjacent bone. Furthermore, 12 more cases have been reported, ${ }^{7,8}$ but these have the same drawbacks as the series described by Volis et al.

\section{Report of Case}

A 23-year-old man reported swelling and pain associated with teeth 27 and 28 to his general dental practitioner. A presumptive diagnosis of an odontogenic infection was made because of the patient's clinical symptoms of swelling and pain. The patient was started on antibiotics on 2 separate occasions and had 2 separate attempts at incision and drainage performed, with no purulence obtained. Because of the refractory nature of his symptoms, he was referred to an oral and maxillofacial surgeon for evaluation and treatment. A periapical radiograph and an orthopantomogram showed a $1.5-\mathrm{cm}$ lytic lesion displacing the root apices of teeth 27 and 28 (Fig 1). Computed tomogram showed a mixed radiopaque or radiolucent lesion associated with teeth 27 and 28 (Fig 2). On clinical examination, the patient had mobility of teeth 27 and 28 associated with a moderately firm, vestibular swelling smaller than $1 \mathrm{~cm}$ in the same region; there was no evidence of cervical lymphadenopathy. Incisional biopsy was performed on the peripheral extraosseous portion of the lesion associated with teeth 27 and 28 . The incisional biopsy returned a diagnosis of leiomyosarcoma, which was confirmed with positive immunohistochemical stains for smooth muscle actin and desmin. S-100 staining was negative. An increase in mitotic activity, infiltrative growth, cellular anaplasia or pleomorphism, and nuclear hyperchromatism are the features used to distinguish this lesion from a leiomyoma. The patient was then referred to the authors' institution. A metastatic workup was completed, which included computed tomography and positron-emission tomography of the head and neck, chest, abdomen, and pelvis, which were negative except for localization of the intramedullary component of the lesion in the right mandible. There was low-grade hypermetabolic activity on positron-emission tomogram using 18-flurodeoxyglucose as the radioactive isotope, with an SUV of 4.1. The patient's clinical, radiographic, and pathologic data were reviewed at the University of Minnesota Head and Neck Tumor Board and a recommendation was made for surgical extirpation as the most appropriate initial therapy. Owing to the paucity of literature on the response rates of head and neck leiomyosarcomas to induction chemotherapy or radiotherapy, the patient elected to pursue surgical extirpation with immediate microvascular reconstruction. The patient underwent composite resection of the mandible from teeth 23 to 30, which included a 2-cm margin of normal tissue (Fig 3). Immediate reconstruction was performed with a fibula free flap and application of a reconstruction plate using prefabricated resection guides. The patient developed dehiscence of the incision in the floor of the mouth and returned to the operating room on postoperative days 6 and 7 for wound debridement and oral bleeding, respectively. The dehiscent area was repaired with acellular dermis applied to the soleus component of the fibula flap. The patient was discharged home on postoperative day 17. Nasogastric tube feedings were continued for approximately 4 weeks postoperatively to allow for the dehiscence in the floor of the mouth to remucosalize. Once the healing was complete, the patient was advanced to a full diet. The patient was followed closely monthly for the first year and then every 3 months for the second year. The patient continued to be disease free at 31 months after surgery and is being followed clinically and radiographically for signs of tumor recurrence. 


\section{HISTOPATHOLOGY}

The biopsy specimen consisted of an apparent spindle cell neoplastic process, vascular tissue, fragments of vital bone, extravasated red blood cells, and very significant areas of necrosis. The spindle cell area showed elongated cells with abundant cytoplasm. The nuclei had blunt ends. The cells showed a slight fascicular pattern, with occasional areas having a somewhat stori-form arrangement. The necrotic areas retained a fibrillar pattern devoid of nuclei. Several areas of reactive bone formation were present. Because of the cytologic features, a muscle tumor was highest on the differential diagnosis list. Immunohistochemistry studies were performed to aid in identifying the cell of origin. Smooth muscle actin, muscle-specific actin, and desmin were strongly positive (Fig 4A-C); S-100 was negative; and CD-31 stained small blood vessels throughout the specimen, but was negative in the area of the tumor cells. These results indicated a neoplasm of smooth muscle origin. Although the cells did not show significant malignant characteristics and mitotic activity was very low, because of the clinical behavior and the amount of necrosis in the biopsy specimen, special stains were performed that would indicate the number of actively dividing cells and aid in differentiating a benign from a malignant proliferation (Fig 5A). Ki-67, a cellular marker for cell proliferation, stained $20 \%$ to $30 \%$ of the cells in the viable tumor area, indicating a very high rate of cell turnover. Stains were also performed to show mutation of the p53 tumor suppressor gene (Fig 5B). The staining for p53 was positive for most cells in the viable tumor area. These findings strongly suggested a malignant smooth muscle tumor.

The surgical specimen (Fig 6A, B) measured $2.4 \times 2.1 \times 1.0 \mathrm{~cm}$. The histopathologic features were consistent with a low-grade leiomyosarcoma arising in the mandible with erosion of the buccal cortex. The tumor cells showed increased nuclear hyperchromatism and infiltrative growth. There was fewer than 1 mitosis per 10 high-power fields and there was no evidence of angiolymphatic space invasion. The surgical margins were free of tumor.

\section{Discussion}

Leiomyosarcomas account for approximately $7 \%$ of all soft tissue sarcomas and are exceedingly rare in the oral cavity, because, with the exception of blood vessels and the circumvallate papilla of the tongue, there is a relative lack of smooth muscle in this region. ${ }^{9}$

Leiomyosarcomas usually present as a painless mass with swelling and are usually mistaken for a more common lesion of the oral cavity. This was the case with the present patient in that the swelling was initially thought to be an odontogenic infection. However, the patient presented with pain, which is often not observed. Some studies have indicated a slight gender predilection toward women, but there is no current clear consensus. ${ }^{6}$ A systematic review by Vilos et al ${ }^{6}$ showed that there are 2 peak ages when these tumors are identified, namely 10 to 30 and 60 to 70 years.

Histopathologic diagnosis of a leiomyosarcoma is difficult owing to the similarities with its benign counterpart, leiomyoma. ${ }^{2}$ Histologic features, such as atypia, necrosis, cellularity, and number of mitoses per high-power field, have been used as criteria for malignancy. There is no clear consensus as to the exact number of mitoses required to diagnose leiomyosarcoma. Immunohistochemistry also can be used for diagnosis, which involves 
staining for the presence of desmin, muscle-specific actin, and smooth muscle actin. Longitudinal striations may be seen with a phosphotungstic acid hematoxylin stain. Glycogen usually can be visualized within the cells with a periodic acid-Schiff stain, and the cell cytoplasm appears bright red with a Masson tri-chrome stain. Definitive diagnosis is usually made with a tissue biopsy and immunohistochemistry staining for a-smooth muscle actin and desmins. The a-smooth muscle staining is necessary to differentiate the tumor from fibrosarcoma, malignant schwannoma, and spindle cell carcinoma. Other positive markers used in the literature are muscle-specific actin, actin, vimentin, Pan-K, CAM-52, laminin, and PC10. ${ }^{6}$

It has been proposed by Nikitakis et $\mathrm{al}^{9}$ and Enzinger et al ${ }^{10}$ that smooth muscle tumors represent a continuum in which an arbitrary separation must be made between benign and malignant tumors. Histopathologically, a leiomyosarcoma shows fascicles of spindle cells with centrally placed blunt-ended nuclei that have a cigar shape. Multinucleated giant cells are usually seen and in some cases the cells are more epithelioid and rounded with an eosinophilic or clear cytoplasm. Grading of leiomyosarcomas is performed histologically based on the number of mitoses, cellular atypia, pleomorphisms, or other indicators of invasiveness. A widely used criterion for the definition of an aggressive lesion is the presence of at least 5 mitoses in 10 high-power fields. Much of the existing data suggest that cutaneous leiomyosarcomas presenting in the facial skin and oral cavity are generally lowgrade tumors. ${ }^{11}$ The present patient did show some spindle cells but with very few mitoses ( $<1$ in 10 high-power fields), deeming it a low-grade leiomyosarcoma.

Owing to the small number of reported cases of leiomyosarcoma of the oral cavity, there is little consensus on the optimal treatment for leiomyosarcomas. Surgery is generally the first modality of treatment. Radiation and chemotherapy have been used in some centers, but generally are thought to be ineffective. ${ }^{11}$ These modalities have been reserved as adjuvant therapy for very close or positive margins or recurrent disease. Management of leiomyosarcomas consists of surgical excision of the tumor with wide margins. Surgical margins of 1 to $2 \mathrm{~cm}$ are recommended with intraoperative frozen sections. ${ }^{12}$ Margin status is considered the most important prognostic factor for recurrence and survival.

Leiomyosarcomas involving the skull base or with metastasis at presentation have an overall poor prognosis. The main reason for this is the anatomic locations of these tumors make them very challenging to excise because of the close proximity of vital structures, so achieving negative margins is difficult. Positive margins treated with chemotherapy and radiation therapy have shown no significant changes in patients' overall survival. ${ }^{13,14}$ Involvement of lymph nodes occurs in approximately $10 \%$ to $15 \%$ of cases; therefore, elective neck dissection is not recommended. The present patient did not receive a neck dissection because of discussions by the authors' multidisciplinary tumor board. The patient also did not receive any postsurgical chemotherapy or radiation therapy owing to the lowgrade status and a widely resected primary tumor with negative margins. Ethunandan et al ${ }^{7}$ reported an overall 5-year survival rate of 55\% for oral cavity leiomyosarcomas, with decreases to $43 \%$ and $19 \%$ for patients with bony involvement and metastasis, respectively.

Radiographic evaluation of a leiomyosarcoma usually shows a mixed radiopaque or radiolucent lesion with a sclerotic or destructive irregular border. The margins are difficult to 
define and can make surgery challenging. Patel et al ${ }^{15}$ showed that findings at diagnostic computed tomography and magnetic resonance imaging are not specific for leiomyosarcoma and usually show a soft tissue mass.

Recurrence of leiomyosarcoma has been reported at approximately $25 \%$ to $40 \%{ }^{12}$; however, a comprehensive review by Ethunandan et $\mathrm{al}^{7}$ showed a local recurrence rate of $34 \%$ for primary oral leiomyosarcomas. Recurrence rates of $42 \%$ for oral cavity and cutaneous lesions, $70 \%$ for paranasal sinus tumors, and $25 \%$ for the larynx have been reported. ${ }^{16,17}$ Distant metastasis to the lungs and bones has been described at $35 \%$ to $38 \%$ for oral cavity tumors based on 64 patients (17\% with sinonasal tumors and $16 \%$ with esophageal tumors). ${ }^{7,17,18}$

Although this particular patient has remained disease free for several years after treatment, patients with higher-grade disease and involvement of the skull base or metastasis may not have such a favorable outcome. Therefore, newer techniques of identification and more systematic reviews are necessary with molecular genetics or markers to better identify these lesions and provide biologic parameters to optimize treatment and patient survival.

\section{References}

1. Gude P, Tisch M, Kraft K, et al. Leiomyosarcoma of the larynx. HNO. 2006; 54:207. (in German), 2006. [PubMed: 15909146]

2. Wertheimer-Hatch L, Hatch GF III, Hatch BSK, et al. Tumors of the oral cavity and pharynx. World J Surg. 2000; 24:395. [PubMed: 10706911]

3. Kim SM, Myoung H, Choung PH, et al. Metastatic leiomyosarcoma in the oral cavity: Case report with protein expression profiles. J Craniomaxillofac Surg. 2009; 37:454. [PubMed: 19664933]

4. Eppsteiner RW, DeYoung BR, Milhem MM, et al. Leiomyosarcoma of the head and neck: A population-based analysis. Arch Otolaryngol Head Neck Surg. 2011; 137:921. [PubMed: 21930982]

5. Izumi K, Maeda T, Cheng J, et al. Primary leiomyosarcoma of the maxilla with regional lymph node metastasis. Report of a case and review of the literature. Oral Surg Oral Med Oral Pathol Oral Radiol Endod. 1995; 80:310. [PubMed: 7489275]

6. Vilos GA, Rapidis AD, Lagogiannis GD, et al. Leiomyosarcomas of the oral tissues: Clinicopathologic analysis of 50 cases. J Oral Maxillofac Surg. 2005; 63:1461. [PubMed: 16182914]

7. Ethunandan M, Stokes C, Higgins B, et al. Primary oral leiomyosarcoma: A clinico-pathologic study and analysis of prognostic factors. Int J Oral Maxillofac Surg. 2007; 36:409. [PubMed: 17395428]

8. Yan B, Li Y, Pan J, et al. Primary oral leiomyosarcoma: A retrospective clinical analysis of 20 cases. Oral Dis. 2010; 16:198. [PubMed: 20374505]

9. Nikitakis NG, Lopes MA, Bailey JS, et al. Oral leiomyosarcoma: Review of the literature and report of two cases with assessment of the prognostic and diagnostic significance of immunohistochemical and molecular markers. Oral Oncol. 2002; 38:201. [PubMed: 11854069]

10. Weiss SW, Langloss JM, Enzinger FM. Value of S-100 protein in the diagnosis of soft tissue tumors with particular reference to benign and malignant Schwann cell tumors. Lab Invest. 1983; 49:299. [PubMed: 6310227]

11. Montgomery E, Goldblum JR, Fisher C. Leiomyosarcoma of the head and neck: A clinicopathological study. Histopathology. 2002; 40:518. [PubMed: 12047762]

12. Marx, RE., Stern, D. Oral and Maxillofacial Pathology: A Rationale for Diagnosis and Treatment. Chicago: Quintessence; 2003.

13. Cocks H, Quraishi M, Morgan D, et al. Leiomyosarcoma of the larynx. Otolaryngol Head Neck Surg. 1999; 121:643. [PubMed: 10547487] 
14. Kuruvilla A, Wenig BM, Humphrey DM, et al. Leiomyosarcoma of the sinonasal tract. A clinicopathologic study of nine cases. Arch Otolaryngol Head Neck Surg. 1990; 116:1278. [PubMed: 2242259]

15. Patel SC, Silbergleit R, Talati SJ. Sarcomas of the head and neck. Top Magn Reson Imaging. 1999; 10:362. [PubMed: 10643880]

16. Barnes, L. Surgical Pathology of the Head and Neck. 2. New York: Marcel Dekker; 2001.

17. Myers, EN. Cancer of the Head and Neck. Philadelphia: WB Saunders; 2003.

18. Thomas S, McGuff HS, Otto RA. Leiomyosarcoma of the larynx. Case report. Ann Otol Rhinol Laryngol. 1999; 108:794. [PubMed: 10453789] 


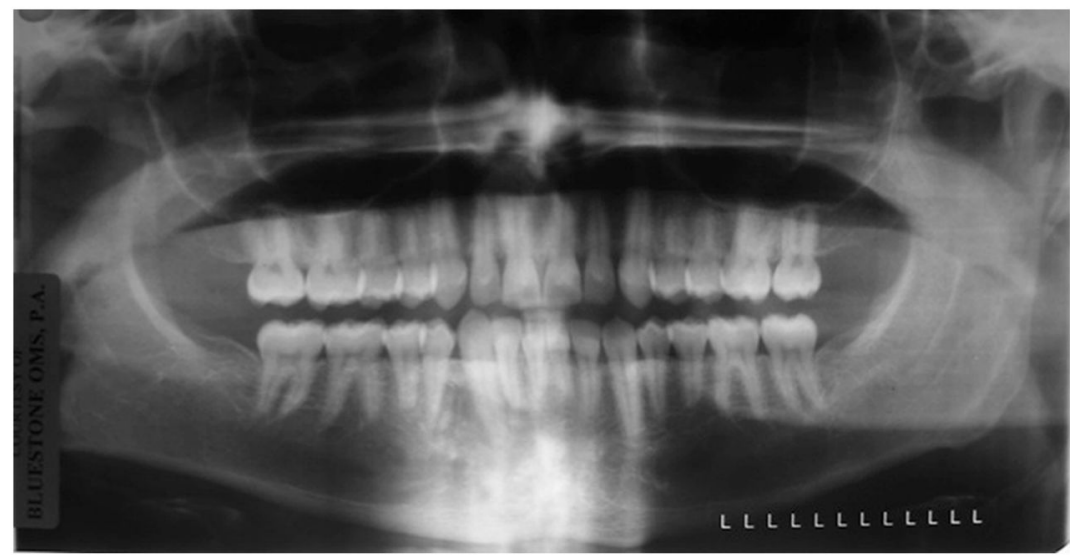

FIGURE 1.

Orthopantomogram showing a mixed radiolucent radiopaque lesion associated with teeth 27 and 28. There was also root divergence noted between the teeth. 


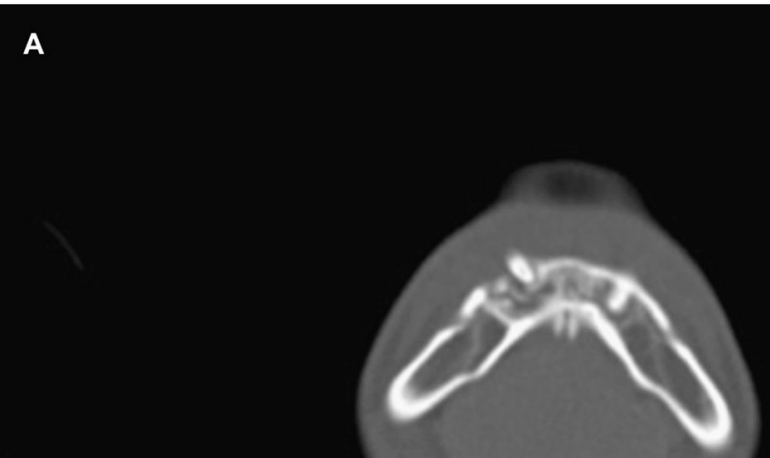


B

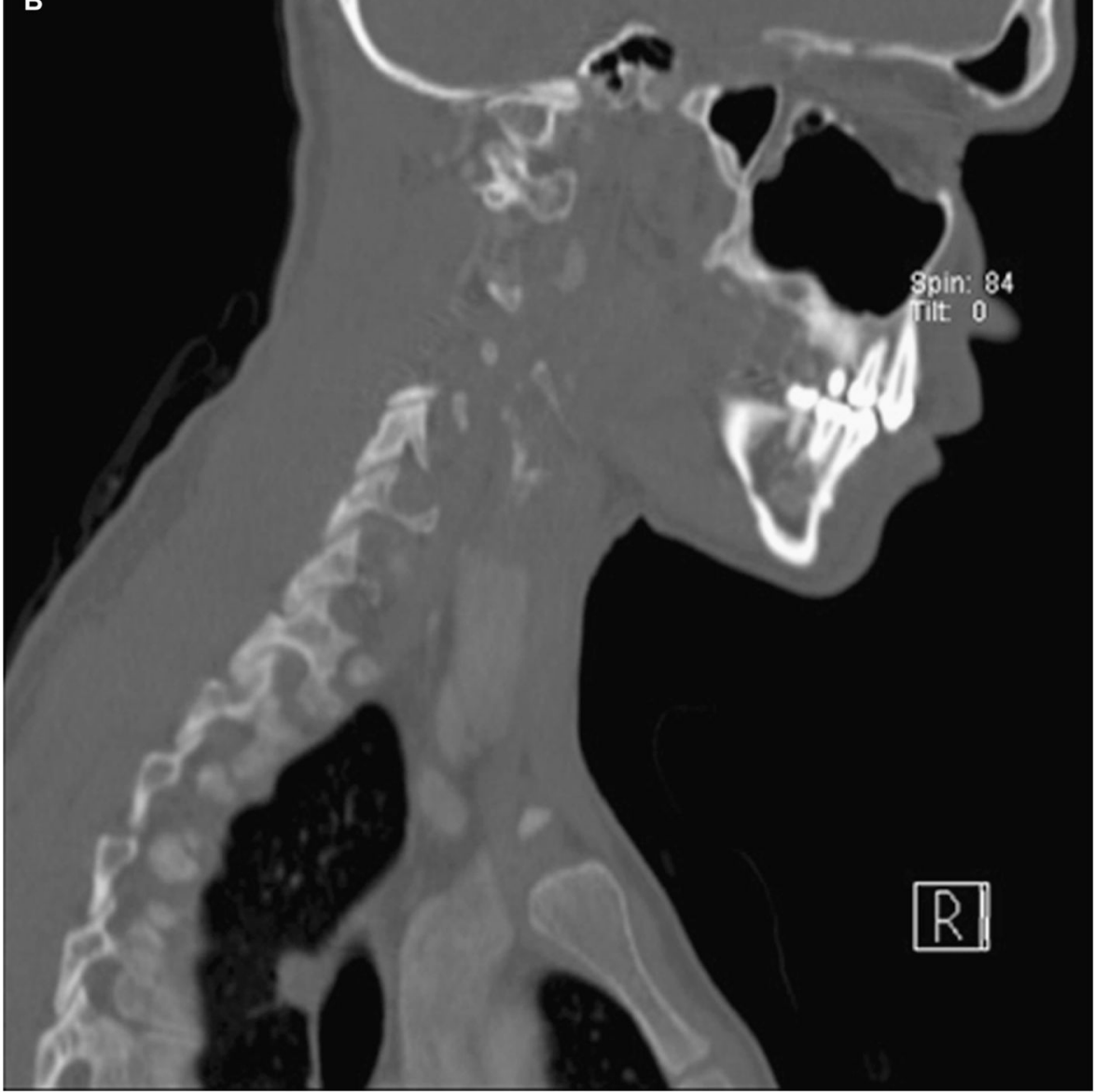

FIGURE 2.

Computed tomograms displaying $A$, axial and $B$, sagittal cuts in a bony window showing the leiomyosarcoma. The images depict a destructive lesion with buccal cortex erosion associated with teeth 27 and 28. 


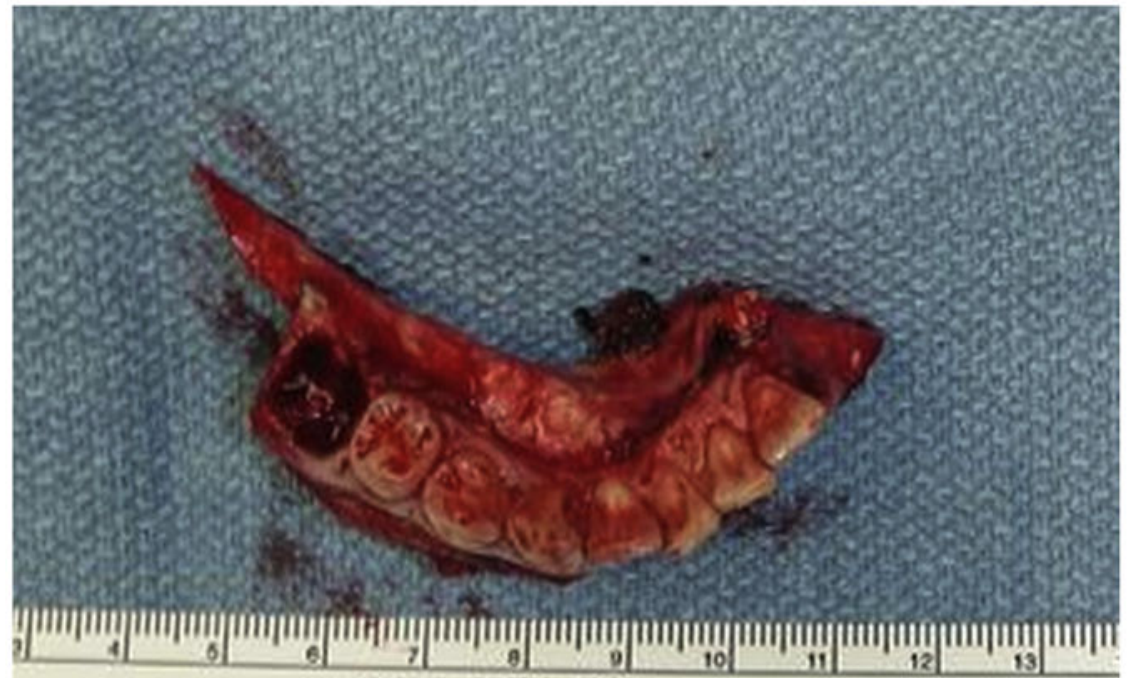

\section{Cardinal Health}

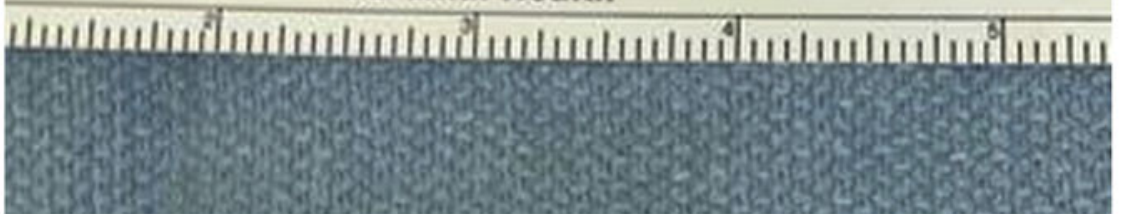

FIGURE 3.

Clinical photograph showing the resected leiomyosar-coma with adequate margins. 

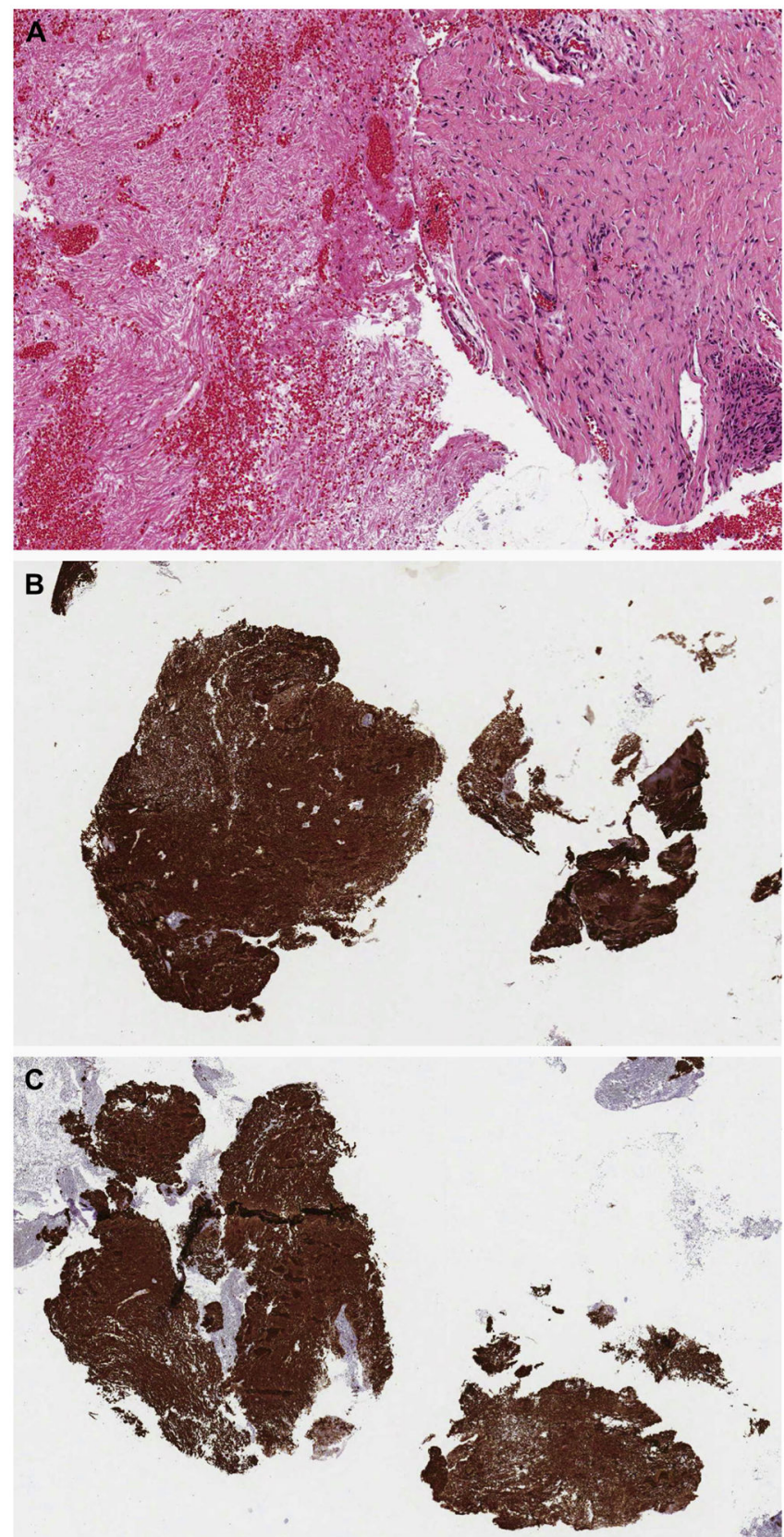

FIGURE 4.

Biopsy specimens. $A$, Spindle cell tumor (right) and necrosis (left). $B$, Muscle-specific actin staining neoplastic cells. $C$, Smooth muscle actin staining neoplastic cells. 



FIGURE 5.

Biopsy specimens. A, Ki-67 staining showing $20 \%$ to $30 \%$ proliferating cells. $B$, P53 staining showing mutation of p53 tumor suppressor gene in most tumor cells. 

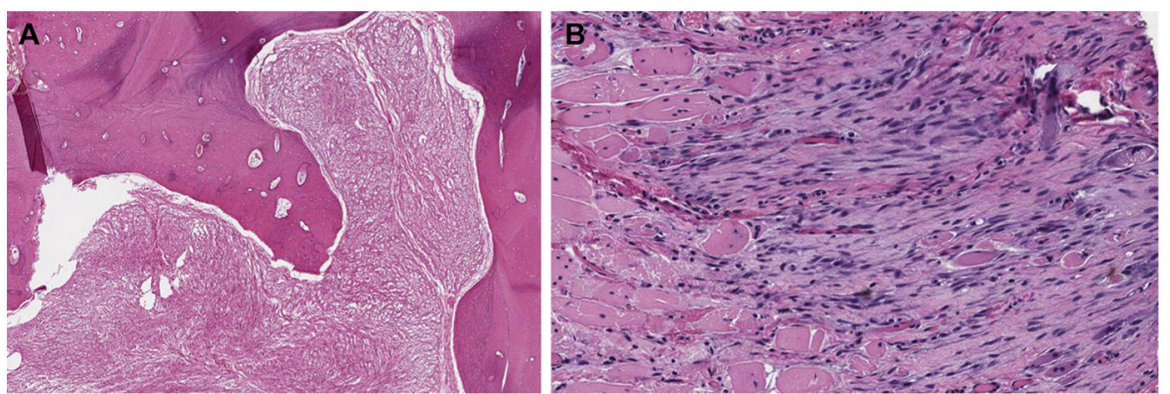

FIGURE 6.

Surgical specimens. A, Area of tumor showing most of the tumor having undergone necrosis. $B$, The malignant tumor has eroded through the cortical plate of the mandible and is invading the skeletal muscle tissue of the mandibular vestibule. 